\title{
Statistical Analysis of the Power Sum of Multiple Correlated Log-Normal Components
}

\author{
Aysel Safak
}

\begin{abstract}
A statistical method is presented for the analysis of the power sum of multiple correlated log-normal random components. The results are compared with those of Schwartz and Yeh and the Monte Carlo simulation. The effect of correlation on the mean and the variance of the combined multiple correlated lognormal components is also investigated. The presented method allows quick and accurate calculations for various system performance parameters of radio networks for high-capacity cellular telephony, two-way paging, packet radio, mobile data networks, and radar detection systems.
\end{abstract}

\section{INTRODUCTION}

$\mathbf{M}$ ULTIPATH with log-normal statistics is important in many areas of radar and communication systems. In some of these areas of interest, such as shadowing in mobile cellular radio systems, these log-normal components may be correlated, causing further complication in the analysis.

For the analysis of the above systems one needs to determine the statistics of the power sum of multipath signals in terms of individual means and variances of the log-normal components. To this end, a number of approximations have been developed in addition to the widespread use of the Monte Carlo simulation [1]-[3]. These approximations have some limitations while the Monte Carlo simulation, though it is more accurate, requires extensive computer time.

The power sum of a finite number of uncorrelated lognormal random variables can be approximated, at least as a first order, by another log-normal probability distribution [4], [5]. This seems to be a good approximation and a close agreement has been observed with the results of the Monte Carlo simulation in the range of 0.1 to 99 percent of the cumulative distribution function [2]. With the above assumption, Schwartz and Yeh developed a recursive procedure to obtain approximations of the mean and variance of the power sum of a number of uncorrelated log-normal variables based on exact expressions for the mean and variance of the power sum of two uncorrelated log-normal random variables [2].

This paper presents an extension to the method of Schwartz and Yeh [2] for the case of correlated log-normal components. Exact analytical formulas are provided for calculating the mean and the variance of the power sum of multiple correlated log-normal components, in contrast with the recursive procedure of Schwartz and Yeh to obtain approximations to

Manuscript received May 8, 1992; revised July 28, 1992

The author is with the Delft University of Technology, Telecommunications and Traffic-Control Systems Group, 2600 GA Delft, The Netherlands

IEEE Log Number 9205013. the mean and variance of the power sum of more than two uncorrelated random variables.

\section{THEORY}

Assume that we want to determine the mean and variance of the power sum of a finite number of variables of the form $\exp \left(y_{i}\right)$ where $y_{i}$ are normally distributed. It is clear that $\exp \left(y_{i}\right)$ has a log-normal probability distribution. The logarithm of the power sum, $s_{k}$, of $k$ log-normally distributed variables of the form $\exp \left(y_{i}\right)$ may be written as

$$
s_{k}=\ln \left(\sum_{i=1}^{k} e^{y_{i}}\right)=\ln \left(e^{s_{k-1}}+e^{y_{k}}\right)=s_{k-1}+\ln \left(1+e^{w_{k}}\right)
$$

where

$$
w_{k}=y_{k}-s_{k-1}
$$

Note that (1) lends itself to an iterative procedure by allowing one to find the power sum of $k$ log-normal components in terms of the power sum of $k-1$ components.

For the sake of convenience, the power sum in (1) is expressed in natural logarithms, though it is generally measured in decibels. Note that a log-normal component $x$ may be written as $10^{x / 10}$ when it is measured in decibels and as $\exp (y)$ when $y$ is expressed in natural logarithms. Then, the relationship between $y$ and $x$ simply becomes

$$
y=\alpha x
$$

where $\alpha=\ln 10 / 10=0.23026$. Similarly, when the mean $m_{x}$, and standard deviation, $\sigma_{x}$, of $x$ are given in decibels they should be multiplied by $\alpha$ in order to find the corresponding values of the mean and standard deviation of $y$.

Here, we will be interested only in the mean and variance of $s_{k}$. By using the last term in (1), the mean value of $s_{k}, E\left(s_{k}\right)$, may be written as

$$
m_{s_{k}}=E\left(s_{k}\right)=m_{s_{k-1}}+G_{1}\left(\sigma_{w_{k}}, m_{w_{k}}\right)
$$

where mean value and variance of $w_{k}$ are found from (2) as follows:

$$
m_{w_{k}}=E\left(w_{k}\right)=m_{y_{k}}-m_{s_{k-1}}
$$

$$
\sigma_{w_{k}}^{2}=E\left(w_{k}^{2}\right)-m_{w_{k}}^{2}=\sigma_{y_{k}}^{2}+\sigma_{s_{k-1}}^{2}-2 \rho_{s_{k-1}, y_{k}} \sigma_{y_{k}} \sigma_{s_{k-1}} .
$$


$\rho_{x, y}$ is defined as the correlation coefficient between $x$ and $y$ :

$$
\rho_{x, y}=\frac{E\left(\left[x-m_{x}\right]\left[y-m_{y}\right]\right)}{\sigma_{x} \sigma_{y}}
$$

and

$$
G_{1}\left(\sigma_{w_{k}}, m_{w_{k}}\right)=E\left[\ln \left(1+e^{w_{k}}\right)\right] .
$$

An analytical expression for (8) is given by (A1) in Appendix A.

The variance of $s_{k}$ is defined by

$$
\sigma_{s_{k}}^{2}=E\left(s_{k}^{2}\right)-m_{s_{k}}^{2} .
$$

Inserting (1) into (9), one obtains

$$
\begin{aligned}
\sigma_{s_{k}}^{2}= & \sigma_{s_{k-1}}^{2}+m_{s_{k-1}}^{2}-m_{s_{k}}^{2}+G_{2}\left(\sigma_{w_{k}}, m_{w_{k}}\right) \\
& +2 E\left[s_{k-1} \ln \left(1+e^{w_{k}}\right)\right]
\end{aligned}
$$

where

$$
G_{2}\left(\sigma_{w_{k}}, m_{w_{k}}\right)=E\left[\ln ^{2}\left(1+e^{w_{k}}\right)\right]
$$

is given by (A2). Calculation of the last term in (10) is presented in Appendix B.

Use of (4), (B4), (B6), and (10) leads to

$$
\begin{aligned}
\sigma_{s_{k}}^{2}= & \sigma_{s_{k-1}}^{2}-G_{1}^{2}\left(\sigma_{w_{k}}, m_{w_{k}}\right)+G_{2}\left(\sigma_{w_{k}}, m_{w_{k}}\right) \\
& +2 \frac{\sigma_{s_{k-1}}}{\sigma_{w_{k}}^{2}}\left(\rho_{s_{k-1}, y_{k}} \sigma_{y_{k}}-\sigma_{s_{k-1}}\right) G_{3}\left(\sigma_{w_{k}}, m_{w_{k}}\right)
\end{aligned}
$$

To determine the mean and variance of the power sum of a number of correlated log-normal variables, one needs to express the correlation coefficient between $s_{k-1}$ and $y_{k}$ in terms of the correlation coefficients between the individual components $y_{i}$. For this purpose, replacing $x$ by $s_{k-1}$ from (1) and $y$ by $y_{k}$ in (7), one gets

$$
\rho_{s_{k-1}, y_{k}}=\rho_{s_{k-1}, y_{k}} \frac{\sigma_{s_{k-2}}}{\sigma_{s_{k-1}}}+\rho_{w_{k-1}, y_{k}} \frac{G_{3}\left(\sigma_{w_{k-1}}, m_{w_{k-1}}\right)}{\sigma_{w_{k-1}} \sigma_{s_{k-1}}} \text {. }
$$

Similarly, substituting $w_{k-1}$ from (2) for $x$ and $y_{k}$ for $y$ into (7) leads to

$$
\rho_{w_{k-1}, y_{k}}=\frac{\rho_{y_{k}, y_{k-1}} \sigma_{y_{k-1}}-\rho_{s_{k-2}, y_{k}} \sigma_{s_{k-2}}}{\sigma_{w_{k-1}}}
$$

Inserting (14) into (13) and rearranging, one obtains

$$
\begin{aligned}
\rho_{s_{k-1}, y_{k}}= & \rho_{s_{k-2}, y_{k}} \frac{\sigma_{s_{k-2}}}{\sigma_{s_{k-1}}}\left(1-\frac{G_{3}\left(\sigma_{w_{k-1}}, m_{w_{k-1}}\right)}{\sigma_{w_{k-1}}^{2}}\right) \\
& +\rho_{y_{i k}, y_{k-1}} \frac{\sigma_{y_{k-1}} G_{3}\left(\sigma_{w_{k-1}, m_{w_{k-1}}}\right)}{\sigma_{s_{k-1}} \sigma_{w_{k-1}}^{2}}
\end{aligned}
$$

One can easily generalize (15) to show that

$$
\begin{aligned}
\rho_{s_{i}, y_{k}}= & \rho_{s_{i-1}, y_{k}} \frac{\sigma_{s_{i-1}}}{\sigma_{s_{i}}}\left(1-\frac{G_{3}\left(\sigma_{w_{i}}, m_{w_{i}}\right)}{\sigma_{w_{i}}^{2}}\right) \\
& +\rho_{y_{i}, y_{k}} \frac{\sigma_{y_{i}}}{\sigma_{s_{i}} \sigma_{w_{i}}^{2}} G_{3}\left(\sigma_{w_{i}}, m_{w_{i}}\right)
\end{aligned}
$$

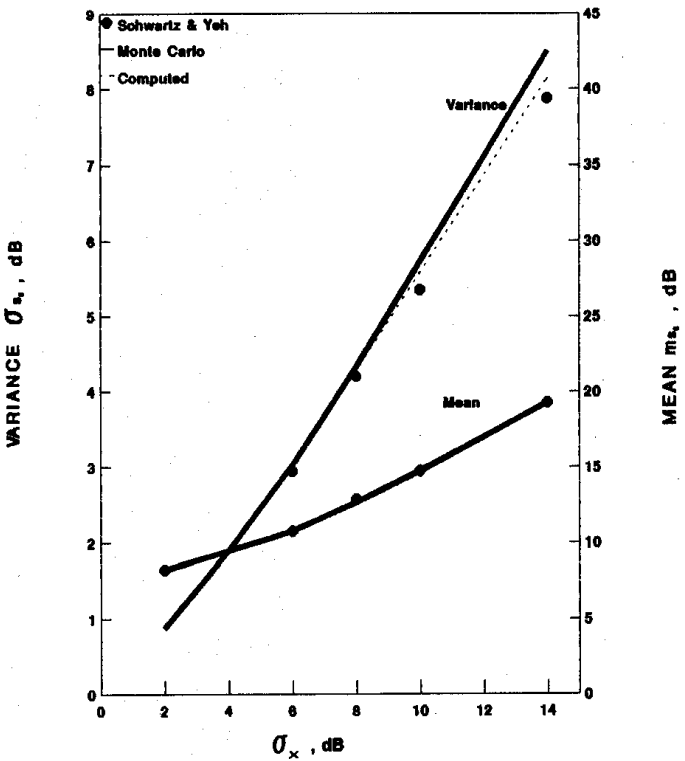

Fig. 1. Mean and variance of the power sum of six log-normal uncorrelated components, each having a mean value of $0 \mathrm{~dB}$, as a function of component variance.

where $i$ varies between 2 and $k-1$. The last two formulas can be used to determine the correlation coefficients between $s_{i}$ and $y_{k}$ which are necessary in order to find the mean and the variance of the sum of $k$ correlated log-normal variables in terms of the correlation coefficients between the individual components $y_{i}$.

\section{RESULtS}

The methodology presented above can be used to find the mean and the variance of the power sum of a number of correlated log-normal components. For the sake of simplicity, in the results to be presented, the components are all assumed to have identical means and variances and the correlation coefficients between individual components are all the same. However, all kinds of combinations of these parameters can be accommodated.

Fig. 1 compares our results with those of Schwartz and Yeh and the Monte Carlo simulation for a decibel spread ranging from 2 to $14 \mathrm{~dB}$ of six uncorrelated log-normal variables, all having $0 \mathrm{~dB}$ means [2]. The number of terms in the summations in (A1)-(A3) are taken 40 even though the series are observed to converge more rapidly for smaller variance values. Fig. 1 shows that the computed results for the mean value of the power sum of six uncorrelated variables agree very closely with those of Monte Carlo and Schwartz and Yeh [2]. As for the variance of the sum, our results agree very closely with the others for the values of the component variances, $\sigma_{x}$, smaller than $8 \mathrm{~dB}$. For larger values of $\sigma_{x}$ our results agree better with the Monte Carlo simulation than those of Schwartz and Yeh. The difference between our results and those of Schwarz and Yeh may be attributed to the fact that Schwartz and Yeh use polynomial approximations to G1, $\mathrm{G} 2$, and $\mathrm{G} 3$ defined by (A1)-(A3) in contrast with exact 


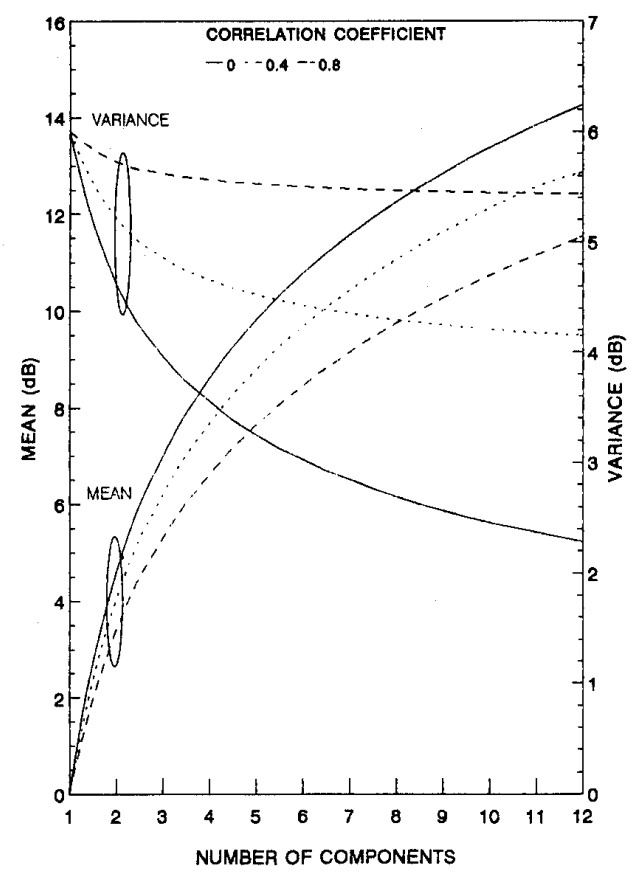

Fig. 2. Mean and variance of the power sum of up to twelve log-normal correlated components, each having a $0-\mathrm{dB}$ mean and a $6-\mathrm{dB}$ component variance, for various values of the correlation coefficients between components.

calculations carried out in this paper. These approximations are reported to differ about 1 percent from the exact calculations directly from (A1)-(A3) [2].

Fig. 2 shows the variation of mean and the variance of the power sum of $k \log$-normal components, given by (4) and (12) respectively, for $k$ ranging from 2 to 12 . The input variables are characterized by $m_{y_{k}}=0 \mathrm{~dB}$ and $\sigma_{y_{k}}=6 \mathrm{~dB}$ for $k=1$ to 12 . Similarly, correlation coefficients between individual components are all assumed to be equal to $0,0.4$, and 0.8 . One can easily observe that increasing correlation between the individual components causes a considerable decrease in the mean but increases the variance of the sum signal. Note that, by virtue of (4), for nonzero mean values of the input variables simply shifts the mean curve shown in Fig. 2. Fig. 3 shows similar results for the sum of up to twelve correlated lognormal variables with a $0-\mathrm{dB}$ mean and a $12-\mathrm{dB}$ variance for various values of the correlation coefficient between individual components.

The variation with $k$ of the correlation coefficient between the $k$ th component and the power sum of $k-1$ components, as given by (15), is plotted in Fig. 4 of identical component means of $0 \mathrm{~dB}$, variance of $6 \mathrm{~dB}$ and $12 \mathrm{~dB}$ and identical correlation coefficient values of 0.4 and 0.8 between the individual components. One can observe that the effect of component variances becomes less for the value of 0.8 of the correlation coefficient as compared to the case of 0.4 .

\section{CONCLUSIONS}

This paper presents a statistical method for analysis of the sum of a finite number of correlated log-normal variables. The

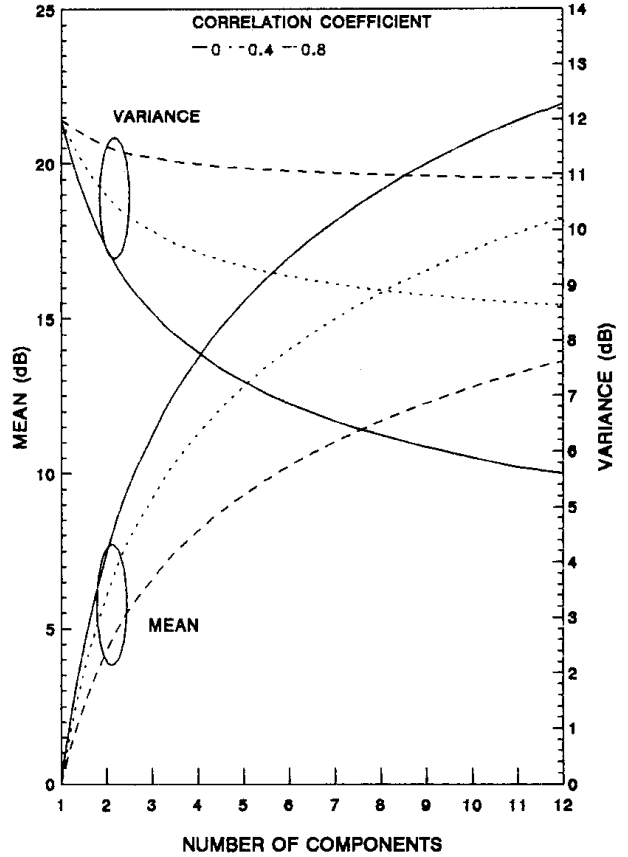

Fig. 3. Mean and variance of the power sum of up to twelve-log-normal correlated components, each having $0-\mathrm{dB}$ mean and a $12-\mathrm{dB}$ component variance, for various values of the correlation coefficients between components.

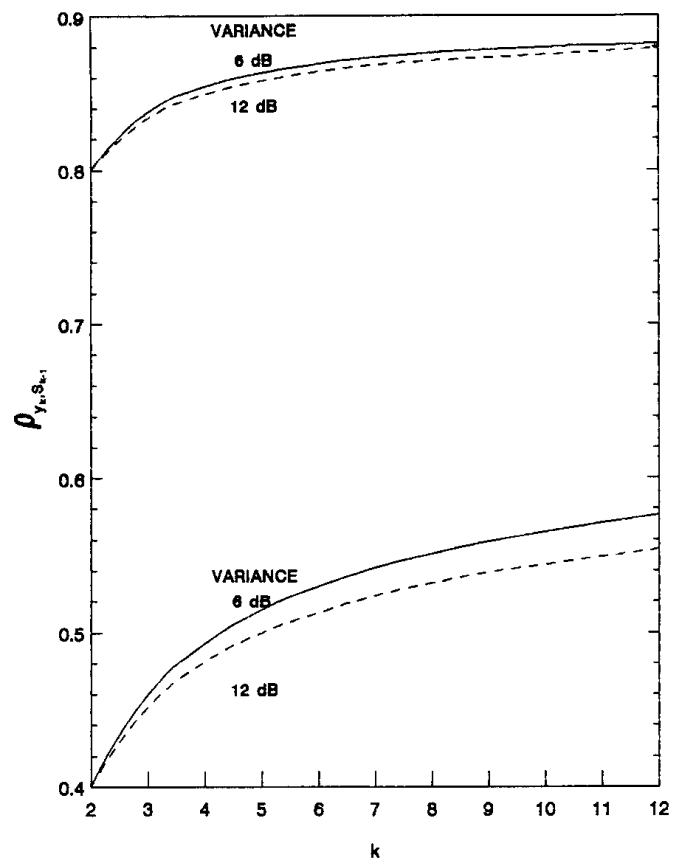

Fig. 4. Variation of (15) with $k$ for component variances of $6 \mathrm{~dB}$ and $12 \mathrm{~dB}$ and values of 0.4 and 0.8 of the correlation coefficient between components.

accuracy of the presented method is established by observing a very close agreement with those of the Monte Carlo simulation for the uncorrelated case. In view of its simplicity and accuracy, this methodology is believed to serve as a useful 
tool in solving some propagation problems with log-normal statistics.

\section{APPENDIX A}

ANALYTICAL EXPRESSIONS FOR $G_{1}, G_{2}$ AND $G_{3}$

The expressions presented below are taken from Schwartz and Yeh [2] with some simplifications and the correction of the sign of the second term in (A1):

$$
\begin{aligned}
G_{1}(\sigma, m)= & m \Phi\left(\frac{m}{\sigma}\right)+\frac{\sigma}{\sqrt{2 \pi}} e^{-\frac{m^{2}}{2 \sigma^{2}}} \\
& +\sum_{k=1}^{\infty} C_{k}[F(\sigma, m, k)+F(\sigma,-m, k)](\mathrm{A} 1) \\
G_{2}(\sigma, m)= & \left(m^{2}+\sigma^{2}\right) \Phi\left(\frac{m}{\sigma}\right)+(m+\ln 4) \frac{\sigma}{\sqrt{2 \pi}} e^{-\frac{m^{2}}{2 \sigma^{2}}} \\
& +2 \sum_{k=1}^{\infty} C_{k}\left(m-k \sigma^{2}\right) F(\sigma, m, k) \\
& +\sum_{k=2}^{\infty} B_{k-1}[F(\sigma, m, k)+F(\sigma,-m, k)] \quad(\mathrm{A} 2) \\
G_{3}(\sigma, m)= & \sigma^{2} \sum_{k=0}^{\infty}(-1)^{k}[F(\sigma, m, k)+F(\sigma,-m, k+1)]
\end{aligned}
$$

where

$$
\begin{gathered}
F(\sigma, m, k)=e^{-k m+\frac{k^{2} \sigma^{2}}{2}} \Phi\left(\frac{m-k \sigma^{2}}{\sigma}\right) \\
\Phi(X)=\frac{1}{\sqrt{2 \pi}} \int_{-\infty}^{x} e^{-\frac{t^{2}}{2}} d t \\
C_{k}=\frac{(-1)^{k+1}}{k} \\
B_{k}=\frac{2(-1)^{k+1}}{k+1} \sum_{j=1}^{k} \frac{1}{j}
\end{gathered}
$$

\section{APPENDIX B}

CALCULATION OF $E\left[s_{k-1} \ln \left(1+e^{w_{k}}\right)\right]$

In view of [6]

$$
E\left[g_{1}(x) g_{2}(y)\right]=E\left[g_{1}(x) g_{2}(y)(x)\right]
$$

one can express

$$
E\left[s_{k-1} \ln \left(1+e^{w_{k}}\right)\right]=E\left[\ln \left(1+e^{w_{k}}\right) E\left(s_{k-1} \mid w_{k}\right)\right] .
$$

The conditional expected value in (B2) may be written as [6]

$$
E\left[s_{k-1} \mid w_{k}\right]=m_{s_{k-1}}+\rho_{s_{k-1}, w_{k}} \frac{\sigma_{s_{k-1}}}{\sigma_{w_{k}}}\left(w_{k}-m_{w_{k}}\right) .
$$

Inserting (B3) into (B2) yields

$$
\begin{aligned}
E\left[s_{k-1} \ln \left(1+e^{w_{k}}\right)\right]= & m_{s_{k-1}} G_{1}\left(\sigma_{w_{k}}, m_{w_{k}}\right) \\
& +\rho_{s_{k-1}, w_{k}} \frac{\sigma_{s_{k-1}}}{\sigma_{w_{k}}} G_{3}\left(\sigma_{w_{k}}, m_{w_{k}}\right)
\end{aligned}
$$

where $G_{1}$ is given by (8) and (A1). (A3) provides an analytical expression for $G_{3}$ which is defined as

$$
G_{3}\left(\sigma_{w_{k}}, m_{w_{k}}\right)=E\left[\left(w_{k}-m_{w_{k}}\right) \ln \left(1+e^{w_{k}}\right)\right] .
$$

By virtue of (2) and (7), the correlation coefficient between $s_{k-1}$ and $w_{k}$ may be expressed in terms of the correlation coefficient between $s_{k-1}$ and $y_{k}$ as follows:

$$
\rho_{s_{k-1}, w_{k}}=\frac{\rho_{s_{k-1}, y_{k}} \sigma_{y_{k}}-\sigma_{s_{k-1}}}{\sigma_{w_{k}}} .
$$

\section{ACKNOWLEDGMENT}

The author would like to thank the anonymous reviewers for their helpful suggestions. The author is also grateful to Prof. Prasad for suggesting the subject and discussing this paper.

\section{REFERENCES}

[1] L. F. Fenton, "The sum of log-normal probability distributions in scatter transmission systems," IRE Trans., vol. CS-8, pp. 57-67, Mar. 1960.

[2] S. C. Schwartz and Y.S. Yeh, "On the distribution function and moments of power sums with log-normal components," Bell Syst. Tech. J., vol. 61, no. 7 , pp. $1441-1462$, Sept. 1982.

[3] M.A. Hamdan, "The logarithm of the sum of two correlated lognormal variates," J. Amer. Statistical Association, vol. 66, no. 333, pp. 105-106, Mar. 1971

[4] W.A. Janos, "Tail of the distribution of sums of log-normal variates," IEEE Trans. Informat. Theory, vol. IT-16, pp. 299-302, May 1970.

[5] P.F. Panter, Communication Systems Design: Line-of-sight and Troposcatter Systems. New York: McGraw Hill, 1972.

[6] D. R. Barr and P.W. Zehna, Probability. CA: Brooks/Cole Publishing Company, 1971

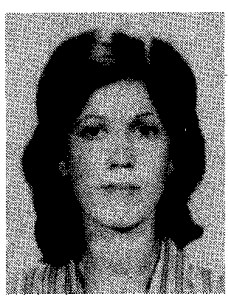

Aysel Safak was born in Uzunköprü, Turkey, in 1952. She received B.Sc. and M.Sc.(Eng.) degrees from Hacettepe University in 1975 and 1977, respectively

From 1977 to 1978 , she worked as an assistant in the Engineering Department of Hacettepe University. Between 1979 and 1984, she was with the Satellite Communications Division of the Turkish PTT Administration in Ankara where she was involved with planning, evaluation, and procurement of analogue and digital earth stations. Currently, she is an associate researcher in the Electrical Engineering Department of the Delft University of Technology, The Netherlands, and is working toward the Ph.D. degree. Her research interests include mobile and indoor communications. 